EPJ Web of Conferences 75, 05007 (2014)

DOI: $10.1051 /$ epjconf/20147505007

(C) Owned by the authors, published by EDP Sciences, 2014

\title{
Non-uniform coupling model of the frustrated chromium-based ring $\mathrm{Cr}_{8} \mathrm{Ni}$
}

\author{
Michał Antkowiak ${ }^{1}$, Łukasz Kucharski ${ }^{1}$, and Grzegorz Kamieniarz ${ }^{1 \mathrm{a}}$ \\ ${ }^{1}$ Department of Physics, A. Mickiewicz University, Poznań, Poland
}

\begin{abstract}
A numerically exact spin-Hamiltonian approach has been proposed for the frustrated $\mathrm{Cr}_{8} \mathrm{Ni}$ molecule. The non-uniform exchange couplings parameters, improving the fit of the experimental magnetic susceptibility data, have been obtained, using a genetic algorithm search procedure. The energy intervals between the lowest multiplets, relevant for envisaged transitions observable in the INS spectra, have been determined and the critical fields corresponding to the first-level crossing have been estimated in agreement with experiment.
\end{abstract}

\section{Introduction}

Molecular nanomagnets based on transition metal ions have been very intensively investigated [1]. Their popularity is mostly due to the fact that quantum phenomena characteristic for a single molecule (like, e.g., quantum tunneling or step like field dependence of magnetization) can be observed in bulk samples. It is possible because nanomolecules are magnetically shielded from each other by organic ligands and the dominant interactions are those within the molecule. There are also expectations that this kind of materials may find application in quantum computing [2-5] and information storage devices [6].

A large family of molecular nanomagnets comprises ring-shaped molecules. Most of them contain even number of antiferromagnetically interacting ions. Only recently the first odd membered antiferromagnetic molecules have been reported [7-11]. They are especially interesting because of magnetic frustration which is expected to appear in this kind of materials.

The frustrated odd-numbered molecule analysed here $\left(\mathrm{C}_{2} \mathrm{H}_{11}\right)_{2} \mathrm{NH}_{2} \mathrm{Cr}_{8} \mathrm{NiF}_{9}\left[\mathrm{O}_{2} \mathrm{CC}\left(\mathrm{CH}_{3}\right)_{3}\right]_{18} \quad\left(\mathrm{Cr}_{8} \mathrm{Ni}\right.$ in short $)$ belongs to the chromium rings family [12]. It was obtained by doping a nickel ion into a ring of eight chromium ions.

Antiferromagnetic interactions between the nearest chromium ions and between chromium and nickel ions (respectively $J=16 \mathrm{~K}$ and $J^{\prime}=70 \mathrm{~K}$, denoted as $C$ ) were initially obtained by fitting the magnetic susceptibility $\chi[7,13]$. The notation is explained in the graphical model shown in Fig. 1. In the calculations the same value of the factor $g=2$ was assumed for both types of ions and the single-ion anisotropy was neglected. Parameters showing better fit to the susceptibility curve were proposed by Furukawa et al. [14] $\left(J=14.7 \mathrm{~K}, J^{\prime}=85 \mathrm{~K}, D_{C r}=-0.42\right.$ $\mathrm{K}, D_{N i}=-4.9 \mathrm{~K}$, denoted as $F$ ) and the role of the local Dzyaloshinskii-Moriya interactionwas was pointed out to explain the step blur in the in-field magnetization curves. This time the realistic values of $g$ were adopted, 1.98 for

\footnotetext{
ae-mail: antekm@amu.edu.pl
}

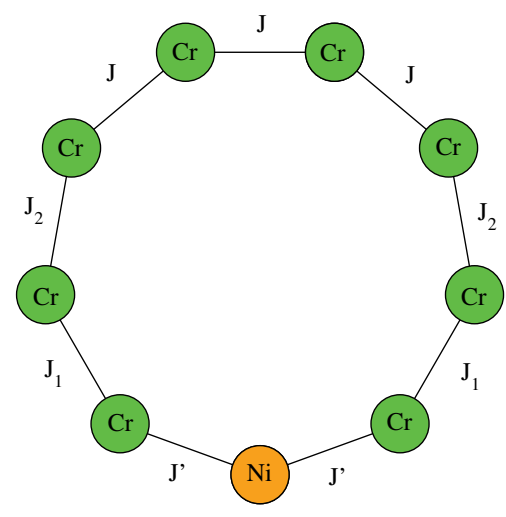

Figure 1. The graphical model of $\mathrm{Cr}_{8} \mathrm{Ni}$ molecule.

chromium and 2.20 for nickel. The single-ion anisotropy was taken into account but on the level of perturbative calculations.

In both approaches the ratio $\alpha=J^{\prime} / J$ is surprisingly high, as for the non-frustrated molecule $\mathrm{Cr}_{7} \mathrm{Ni}[15,16]$ it is much smaller. The dramatic jump in the magnetic coupling $J^{\prime}$ may be an artifact of the oversimplified model so that we aim at finding a non-uniform coupling model, lowering the value of $\alpha$ and improving agreement with experiment.

\section{Models and methods applied}

The model of $\mathrm{Cr}_{8} \mathrm{Ni}$ molecule is presented on Fig. 1 and is described by the following Heisenberg Hamiltonian:

$$
\mathcal{H}=\sum_{j=1}^{8} J_{j} \mathbf{S}_{j} \cdot \mathbf{S}_{j+1}+\sum_{j=1}^{9}\left(D_{j}\left(S_{j}^{z}\right)^{2}-g_{j} \mu_{B} \mathbf{B} \cdot \mathbf{S}_{j}\right),
$$

where the Ni ion with spin $S=1$ is located at site $j=1$.

We consider models with varying degree of nonhomogeneity of exchange integrals between chromium 


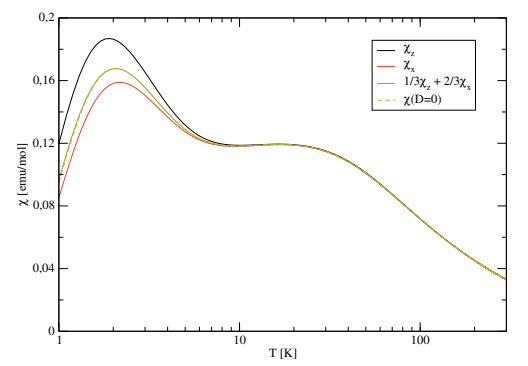

Figure 2. Temperature dependence of magnetic susceptibility $\chi_{x}, \chi_{z}$ and averaged susceptibility (green curve) calculated for $\mathrm{Cr}_{6} \mathrm{Ni}$ model with parameters of model $F$. The latter is compared to susceptibility $\chi(D=0)$ of the corresponding isotropic model.

ions (Fig. 1). For all models the value of the coupling between the chromium and nickel ions is denoted by $J^{\prime}$, the value of the exchange integral between the chromium ions closest to the nickel ion is denoted by $J_{1}$ and the next in the order by $J_{2}$ (see Fig. 1). Other couplings between the chromium ions are denoted by $J$.

The basic model, which we call $2 J$, is characterized by equal values of the couplings between the ions of chromium $J$, therefore $J_{1}=J_{2}=J$. Models proposed by Cador et al. [7, 13] $C$ and Furukawa et al. [14] $F$ are variants of the model $2 J$. The $3 J$ model is characterised by $J_{2}=J$. In the last proposed non-uniform model $4 J$ the values $J^{\prime}, J_{1}, J_{2}$ can be different from $J$.

To obtain the numerically precise values of the magnetic properties of the model we use the exact diagonalization (ED) technique. As the experimental data were obtained on a polycrystalline sample, it is important to average the susceptibility in both the $z$ and $x$ direction $\left(\chi=\frac{1}{3} \chi_{z}+\frac{2}{3} \chi_{x}\right)$. However, the calculations of the magnetic properties while the magnetic field is applied in $x$ direction are too demanding with respect to amount of time and memory size, we have tried to simplify the model by neglecting anisotropy. As expected, our calculations for systems with a reduced number of chromium ions $\left(\mathrm{Cr}_{6} \mathrm{Ni}\right.$, $\mathrm{Cr}_{4} \mathrm{Ni}$ ) showed a negligible impact of anisotropy on the averaged magnetic susceptibility due to the relatively low value of anisotropy for chromium and nickel. In the range of temperature for which experimental results have been obtained for $\mathrm{Cr}_{8} \mathrm{Ni}[7,13]$, the susceptibility curve of $\mathrm{Cr}_{6} \mathrm{Ni}$ theoretical ring for zero anisotropy practically coincides with that calculated for a non-zero anisotropy and then averaged, as shown in Fig. 2. The susceptibility curves in the $x$ and $z$ directions are distinct but, the averaged curve and the curve for zero anisotropy coincide. Using this feature we can omit the $x$ direction calculations and get exact results in the isotropic limit, exploiting the ED method.

By skipping the anisotropy we have performed simulations within the non-uniform models $2 J, 3 J$ and $4 J$. In these models the number of parameters is increased and the single susceptibility fit may be ambiguous as far as the parameters are concerned. To estimate their values we have used a genetic algorithm (GA) approach based on the floating-point representation and standard arithmetic ge-
Table 1. The list of the coupling parameters found for different models and the corresponding critical fields $B_{c}$.

\begin{tabular}{|c|ccccc|}
\hline Model & $J[\mathrm{~K}]$ & $J_{2}[\mathrm{~K}]$ & $J_{1}[\mathrm{~K}]$ & $J^{\prime}[\mathrm{K}]$ & $B_{c}[\mathrm{~T}]$ \\
\hline$C$ & 16.00 & $J$ & $J$ & 70.00 & 2.89 \\
$F$ & 14.70 & $J$ & $J$ & 85.00 & 2.72 \\
$2 J$ & 14.38 & $J$ & $J$ & 64.76 & 2.75 \\
$3 J$ & 14.89 & $J$ & 14.15 & 48.66 & 2.67 \\
$4 J$ & 13.55 & 15.23 & 15.75 & 46.20 & 2.67 \\
\hline
\end{tabular}

netic operators. We have minimised the mean squared error defined as:

$$
M S E=\frac{1}{N} \sum_{i=1}^{N}\left(c_{i}-e_{i}\right)^{2},
$$

where $e_{i}$ is $i$-th value obtained experimentally, $c_{i}$ is the corresponding value calculated for a given model, $N$ is the number of experimental points.

GA is a method inspired by natural evolution that has become a commonly used tool to solve multi-objective optimization problems $[17,18]$. It is very robust and works particularly well when the search space is broad and has complex landscape. It works by evaluating a set of potential solutions called a population. Each individual is assigned a value called fitness which is a measure of how well they fit the actual solution. In our case the fitness is the inverse of the $M S E$ value (2). The better the fitness of an individual is, the higher chance of providing more copies into the offspring population it has during a selection operation. After this step the individuals are also additionally mixed with each other by means of cross-over operator and individually via mutation operator. These steps make up a full iteration called a generation.

The initial population was created from a $P=18$ copies of a starting individual (parameters set). The algorithm worked for a fixed number of $G=10000$ generations. As a probabilistic method, GA has the ability to retrieve not only global extrema but local ones too. Moreover, it allows to escape from the attraction area of starting point local extremum, giving access to other possibly better solutions.

\section{Results}

We confirmed the temperature dependence of the susceptibility for the model $C$ [7, 13] and $F$ [14]. As to our model $2 J$, it has improved the quality of the susceptibility fit for the higher temperatures range compared to the $C$ and $F$ model predictions, however for the lowest temperatures it is slightly inferior (inset in Fig. 3) than that of model $F$. Significant improvement in the quality of fit we obtained using the $3 J$ model (red curve in Fig. 3). Model $4 J$ is characterized by an even smaller $M S E$ value, however the susceptibility values are very close to those of $3 J$ model. For the sake of the clarity we do not present the $4 J$ model results on Fig. 3.

Using the parameters obtained for all the models and quoted in Table 1, we calculated the magnetization 


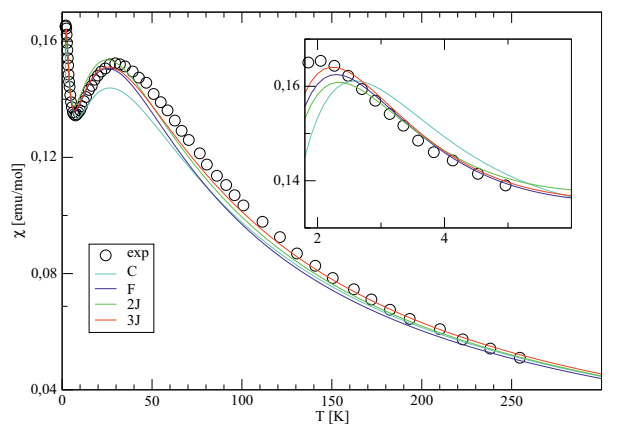

Figure 3. Temperature dependence of susceptibility for different models of $\mathrm{Cr}_{8} \mathrm{Ni}$ molecule. Curves are specified in the legend. Symbols represent experimental data measured for a powder sample.
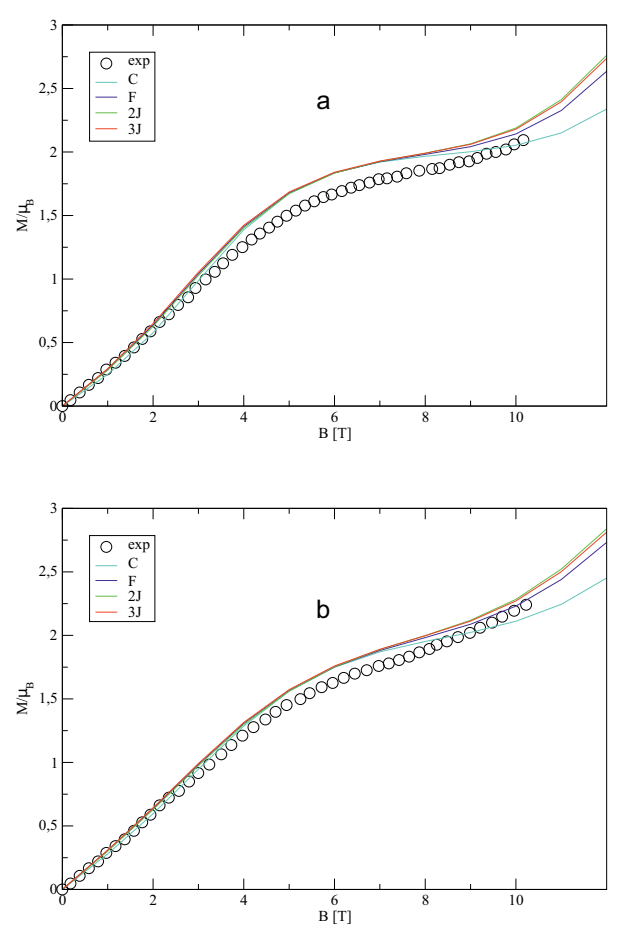

Figure 4. Magnetization of $\mathrm{Cr}_{8} \mathrm{Ni}$ for temperature $T=1.6 \mathrm{~K}$ (panel a) and $T=2 \mathrm{~K}$ (panel b). Curves are specified in the legend. Symbols represent experimental data.

isotherms for $T=1.6 \mathrm{~K}$ and $T=2.0 \mathrm{~K}$ (see Fig. 4). The experimental data were published earlier [7], but have not yet been taken into account in modelling so that the theoretical curves $C$ and $F$ are new as far as we know. Models $F, 2 J$ and $3 J$ lead to quantitative compliance of magnetization curve with experiment in the range of weak magnetic field. In the intermediate range deviations occur and reach $10 \%$. In strong fields exceeding 9T, an improvement of a compatibility with the experimental data can be seen for temperature $T=2 \mathrm{~K}$.

We have also estimated the critical field $B_{c}$ corresponding to the first singlet-triplet level crossing (see Tab. 1). They are consistent with experiment [14] showing the first step in the magnetization profile at $B_{c}=2.8 \mathrm{~T}$ and the on-

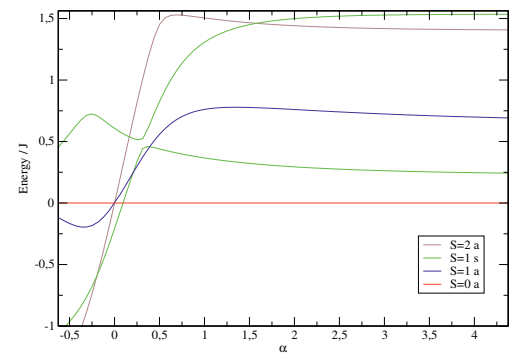

Figure 5. Energy of the lowest states for $2 J$ model as a function of $\alpha=J^{\prime} / J$. The labels $a, s$ in the legend denote the symmetry of the eigenstates with respect to the mirror reflection.

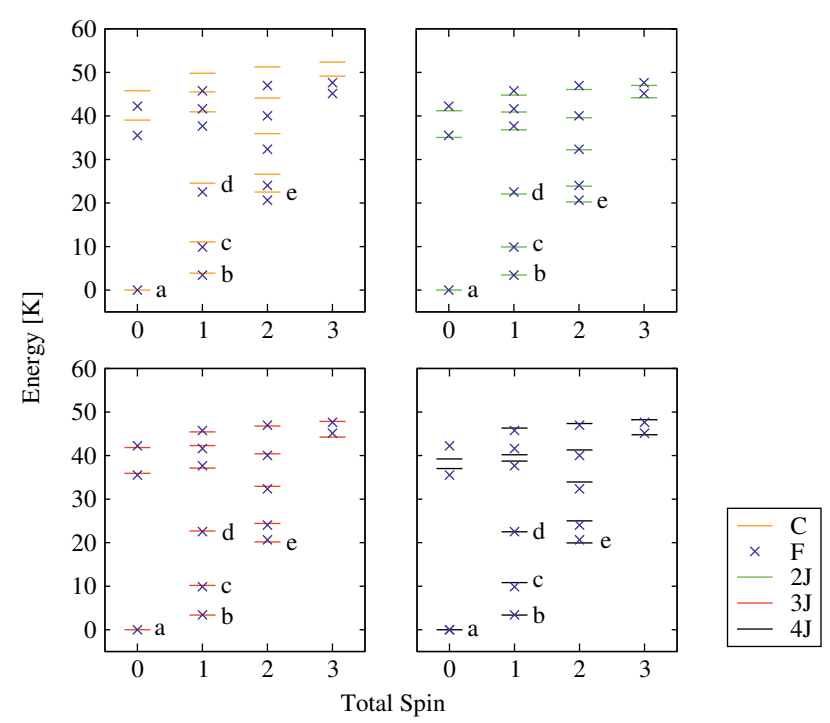

Figure 6. Diagram of the lowest energy levels for different models of $\mathrm{Cr}_{8} \mathrm{Ni}$ molecule. The crosses on every panel represent the predictions of the $F$ model. The lowest five levels are marked by letters $a, b, \ldots, e$.

set of inflection point in Fig. 4a for $T=1.6 \mathrm{~K}$. However, they cannot settle the matter of the best model.

The impact of the ratio $\alpha$ of coupling between the ions of chromium and chromium and nickel ions on the lowenergy structure is presented in Fig. 5 for the $2 J$ model. For $\alpha_{1}<-0,19$ the ground state is the state of $S=2$, for $\alpha_{2}>0.09$ - state $S=0$, whereas in between, the state $S=$ 1. These results agree qualitatively with those obtained earlier [7]. However, the fact that the state $S=1$ is the ground state in the range $\alpha_{1} \leq \alpha \leq \alpha_{2}$ of finite width denies the statement [7] that the ring $\mathrm{Cr}_{8} \mathrm{Ni}$ is not frustrated only for $\alpha=0$. To fully determine the nature of this state it would be necessary to calculate the frustration signatures [11, 19].

Taking into account the parameters given in Table 1, the energy structure diagram for all the models considered is plotted in Fig. 6, whereas in Table 2 we compare the energy differences $\Delta_{\alpha \beta}$ for some transitions allowed by the selection rules [10] which can be probably observed in the inelastic neutron scattering (INS) experiment. The subscripts $\alpha, \beta$ refer to the levels which are identified by the 
Table 2. Energy differences of four lowest lying excited states for different models of $\mathrm{Cr}_{8} \mathrm{Ni}$ molecule relevant for INS experiment. All values in meV.

\begin{tabular}{|c|cccc|}
\hline Model & $\Delta_{a b}$ & $\Delta_{a c}$ & $\Delta_{a d}$ & $\Delta_{b e}$ \\
\hline$C$ & 0.34 & 0.95 & 2.12 & 1.61 \\
$F$ & 0.30 & 0.85 & 1.94 & 1.48 \\
$2 J$ & 0.30 & 0.85 & 1.90 & 1.45 \\
$3 J$ & 0.29 & 0.88 & 1.95 & 1.45 \\
$4 J$ & 0.29 & 0.93 & 1.94 & 1.43 \\
\hline
\end{tabular}

letters $a, b, \ldots, e$ and are shown in Fig. 6 . The values of $\Delta_{\alpha \beta}$ for $C$ model definitely differ from those of other models. For the remaining models, the predictions are similar, although $\Delta_{a c}$ for the $4 J$ model is significantly higher. These values may help in discrimination of the best model, when the INS experiment is performed.

In conclusion, we have established that the nonuniform coupling models improve the susceptibility fit to experiment and provide consistent agreement with the measured magnetization isotherms, including the critical fields $B_{c}$. They reduce the ratio $\alpha$, but not significantly so that they confirm the substantial difference between the coupling $J^{\prime}$ and $J$. These findings were accomplished, optimising the $M S E$ (2), using the genetic algorithm procedure.

We demonstrated that the variant $C$ of the $2 J$ model underestimates the susceptibility results and yields energy intervals significantly different than those following from the remaining models. We expect that further verification of the applicability of the non-uniform models can be provided by INS measurement. In particular, the experimental energy transfer corresponding to the INS peak around $0.9 \mathrm{meV}$ would be useful. It would also be interesting to have the zero-field splittings provided by the EPR transitions. However, to determine these effects, the anisotropy in the model (1) needs to be taken into account.

The calculations were performed on computer facilities granted by PSNC (Poland) as well as within DECI programme by the PRACE-2IP (FP7/2007-2013) under grant agreement no RI-283493. Support from the Polish MNiSW through the grant No N519 579138 is also acknowledged.

One of us (MA) would like to thank Prof. Marc Drillon and Dr. Pierre Rabu for inspiring discussions related to the present topic and warm hospitality during the internship in IPCMS Strasbourg.

\section{References}

[1] D. Gatteschi, R. Sessoli, J. Villain, Molecular nanomagnets (Oxford University Press, Oxford, 2006)
[2] A. Ardavan, O. Rival, J. Morton, S. Blundell, A. Tyryshkin, G. Timco, R. Winpenny, Phys. Rev. Lett. 98, 057201 (2007)

[3] J. Lehmann, A. Gaita-Ariño, E. Coronado, D. Loss, Nature Nanotech. 2, 312 (2007)

[4] G. Timco, S. Carretta, F. Troiani, F. Tuna, R. Pritchard, C. Muryn, E. McInnes, A. Ghirri, A. Candini, P. Santini et al., Nature Nanotech. 4, 173 (2009)

[5] B. Georgeot, F. Mila, Phys. Rev. Lett. 104, 200502 (2010)

[6] M. Mannini, F. Pineider, P. Sainctavit, C. Danieli, E. Otero, C. Sciancalepore, A. Talarico, M.A. Arrio, A. Cornia, D. Gatteschi et al., Nature Mat. 8, 194 (2009)

[7] O. Cador, D. Gatteschi, R. Sessoli, A.L. Barra, G. Timco, R. Winpenny, J. Magn. Magn. Mater. 290291, 55 (2005)

[8] H. Yao, J. Wang, Y. Ma, O. Waldmann, W. Du, Y. Song, Y. Li, L. Zheng, S. Decurtins, X. Xin, Chem. Commun. 16, 1745 (2006)

[9] N. Hoshino, M. Nakano, H. Nojiri, W. Wernsdorfer, H. Oshio, J. Am. Chem. Soc. 131, 15100 (2009)

[10] M.L. Baker, G.a. Timco, S. Piligkos, J.S. Mathieson, H. Mutka, F. Tuna, P. Kozlowski, M. Antkowiak, T. Guidi, T. Gupta et al., Proceedings of the National Academy of Sciences of the United States of America 109, 19113 (2012)

[11] M. Antkowiak, P. Kozłowski, G. Kamieniarz, G.a. Timco, F. Tuna, R.E.P. Winpenny, Physical Review B 87, 184430 (2013)

[12] E.J.L. McInnes, S. Piligkos, G. Timco, R. Winpenny, Coord. Chem. Rev. 249, 2577 (2005)

[13] O. Cador, D. Gatteschi, R. Sessoli, F. Larsen, J. Overgaad, A.L. Barra, S. Teat, G. Timco, R. Winpenny, Angew. Chem., Int. Ed. 43, 5196 (2004)

[14] Y. Furukawa, K. Kiuchi, K. Kumagai, Y. Ajiro, Y. Narumi, M. Iwaki, K. Kindo, A. Bianchi, S. Carretta, P. Santini et al., Phys. Rev. B 79, 134416 (2009)

[15] R. Caciuffo, T.G.G. Amoretti, S. Carretta, E. Liviotti, P. Santini, C. Mondelli, G. Timco, C. Muryn, R. Winpenny, Phys. Rev. B. 71, 174407 (2005)

[16] S. Piligkos, H. Weihe, E. Bill, F. Neese, H.E. Mkami, G. Smith, D. Collison, G. Rajaraman, G. Timco, R. Winpenny et al., Chem. Eur. J. 15, 3152 (2009)

[17] J. Holland, Adaptation in Natural and Artificial Systems: An Introductory Analysis with Applications to Biology, Control and Artificial Intelligence (MIT Press, Cambridge, MA, USA, 1992)

[18] P. Sobczak, Ł. Kucharski, G. Kamieniarz, Comp. Phys. Comm. 182, 1900 (2011)

[19] P. Kozłowski, M. Antkowiak, G. Kamieniarz, Journal of Nanoparticle Research 13, 6093 (2011) 